\title{
Computer-Aided Design and Fabrication of a pediatric Fox Plan in PLA: A Case Report
}

\author{
Nidal Elmoutawakkil1*, Khadija Oumensour ${ }^{2}$ and Samira Bellemkhannate ${ }^{3}$ \\ ${ }_{1,3}^{1,3}$ Department of Removable Dentures at the Ibn Rochd Dental Consultation and Treatment Center in Casablanca, Faculty of Dental medicine Hassan II \\ university, Moracco
}

${ }^{2}$ Department of Oedodontics and Prevention at the Ibn Rochd Dental Consultation and Treatment Center in Casablanca, Faculty of Dental medicine Hassan II university,

Submission: February 08, 2020; Published: February 17, 2020

*Corresponding author: Nidal Elmoutawakkil, Department of Removable Dentures at the Ibn Rochd Dental Consultation and Treatment Center in Casablanca, Faculty of Dental medicine Hassan II university, Moracco

\section{Abstract}

Auto-immune lymphoproliferative syndrome (ALPS) is benign, early-onset (on average before five years), combines multifocal lymphadenopathy, splenomegaly and possibly hepatomegaly; This multi-organ involvement is reflected by an alteration of all the manducatory functions of the affected patients, but xerostomia, immunosuppression, and hypofibrinogenemia reduce the quality of osteomucosal bearing surfaces; in addition to the low salivary viscosity, retention and prosthetic stability become a real challenge. In some more complex cases, where age (13years) and microstomia added to the arsenal of challenges, it becomes necessary to adapt the instruments to reduced pediatric dimensions. The objective of this work is to demonstrate through a clinical case the prosthetic management of a patient suffering from this syndrome, through the computer-aided design and manufacturing of an individualized Fox plan according to patient's proportions made in PLA (polylactic acid) to adjust the occlusion plane appropriately.

Keywords: Auto-immune lymphoproliferative syndrome (ALPS); Neutropenia; Fox plan; CAD-CAM

Abbreviations: ALPS: Autoimmune Lymphoproliferative Syndrome; PLA: Polylactic Acid; VPRD: Vertical Physiological Rest Dimension; CR: Centric Relation

\section{Introduction}

Autoimmune lymphoproliferative syndrome (ALPS) is characterized by immune dysregulation due to a lack of lymphocyte apoptosis. Clinical manifestations may be noted among several siblings and include lymphadenopathy, splenomegaly, increased risk of lymphoma, and autoimmune disease, which generally involve hematopoietic cell lines manifesting as multi-lineage cytopenias. The inherited genetic abnormality of many ALPS patients has involved the transmission pathway signaling proteins (FAS), however, there are still cases where genetic abnormalities are undefined. Biologically, ALPS demonstrates an expansion of T cells that express the alpha/beta T cell receptor but lack CD4 and CD8 in blood and peripheral tissue samples (double-negative T cells, DNT). Other typical laboratory results include high levels of interleukin-10, vitamin B12, and Fas-induced defective apoptosis in vitro. So far, we note that 500 individuals belonging to 300 families have been identified with ALPS [1].

There are several types of ALPS syndrome: i. $\quad$ Type 0 with significant lymphocyte proliferation, starts very soon after birth and causes death in the early years. It is due to a homozygous mutation in the Fas receptor gene.

ii. Type Ia, the most common type, begins in the first year of life with typical lymphocyte proliferation that spontaneously improves without treatment. This type originates from heterozygous mutations in the Fas receptor gene.

iii. Type Ia-MS (for somatic mutation) reaches a part of the lymphocyte cells.

iv. Type Ib is linked to mutations in the gene encoding Fas ligand (FasL).

v. Type II corresponds to mutations in the genes of caspase 8 or caspase 10.

vi. Type III includes lymphoproliferative syndromes of unknown cause. 
The diagnosis is based on tests for measuring apoptosis. The patient's lymphocytes are cultured, activated, and then treated with molecules inducing apoptosis (Fas ligand or anti-Fas agonist antibody). These lymphocytes have a greater or lesser resistance to apoptosis. Subsequently, the Fas receptor is sequenced to look for possible mutations. Patients with severe autoimmune hemolytic anemia or autoimmune thrombocytopenia can be treated effectively with corticosteroids or intravenous immunoglobulins. Patients with autoimmune cytopenia refractory to splenectomy or corticosteroid therapy may be treated with intravenous immunoglobulin, ciclosporin A, vincristine, methotrexate, azathioprine, mycophenolate mofetil or rituximab. Although lymphoproliferation in ALPS is usually benign, there is an increased risk of developing Hodgkin's and non-Hodgkin's lymphoma [2].

\section{Case Report}

\section{Patient identification}

A 13-year-old boy addressed to the Department of removable dentures at the Ibn Rochd Dental Consultation and Treatment Center in Casablanca by his former pedodontist working in the same center for an aesthetic rehabilitation, the patient is suffering from severe neutropenia on ALPS, furthermore, the total edentulism of the patient socially handicaps him and led to school abandonment. The patient had one year of ENT paracentesis with an ear drain to treat chronic mastoiditis, as well as several dental extractions (temporary and permanent teeth) following carious process complications and severe bone lysis. The different hemograms performed show periods of disturbances expressed by hypoplasia or even bone marrow suppression accompanied by hypofibrinogenemia, a reduction in hematocrit, hemoglobin, and MCV (Mean Corpuscular Volume). Note that during these phases the patient seems sleepy and extremely asthenic. In general, the patient presents severe hypoacusis, muscular atrophy, growth retardation (he measures $1 \mathrm{~m} 30$ whereas a child of the same age measures on average $1 \mathrm{~m} 62$ ), underdeveloped secondary sexual characteristics, hypertelorism, a large forehead with a malformation of the external lobe of the ears.

\section{Extraoral examination}

The patient presents an ovoid face, the facial symmetry is respected, harmony between the different stages of the face is disturbed by a clear reduction of the height of the lower stage testifying to the collapse of the vertical dimension of occlusion, the nasolabial folds and under the chin are marked. The profile examination demonstrates a concave typology with a wide-open nasolabial angle. The line of the smile is low according to Liébart's classification (Figure 1) [3].

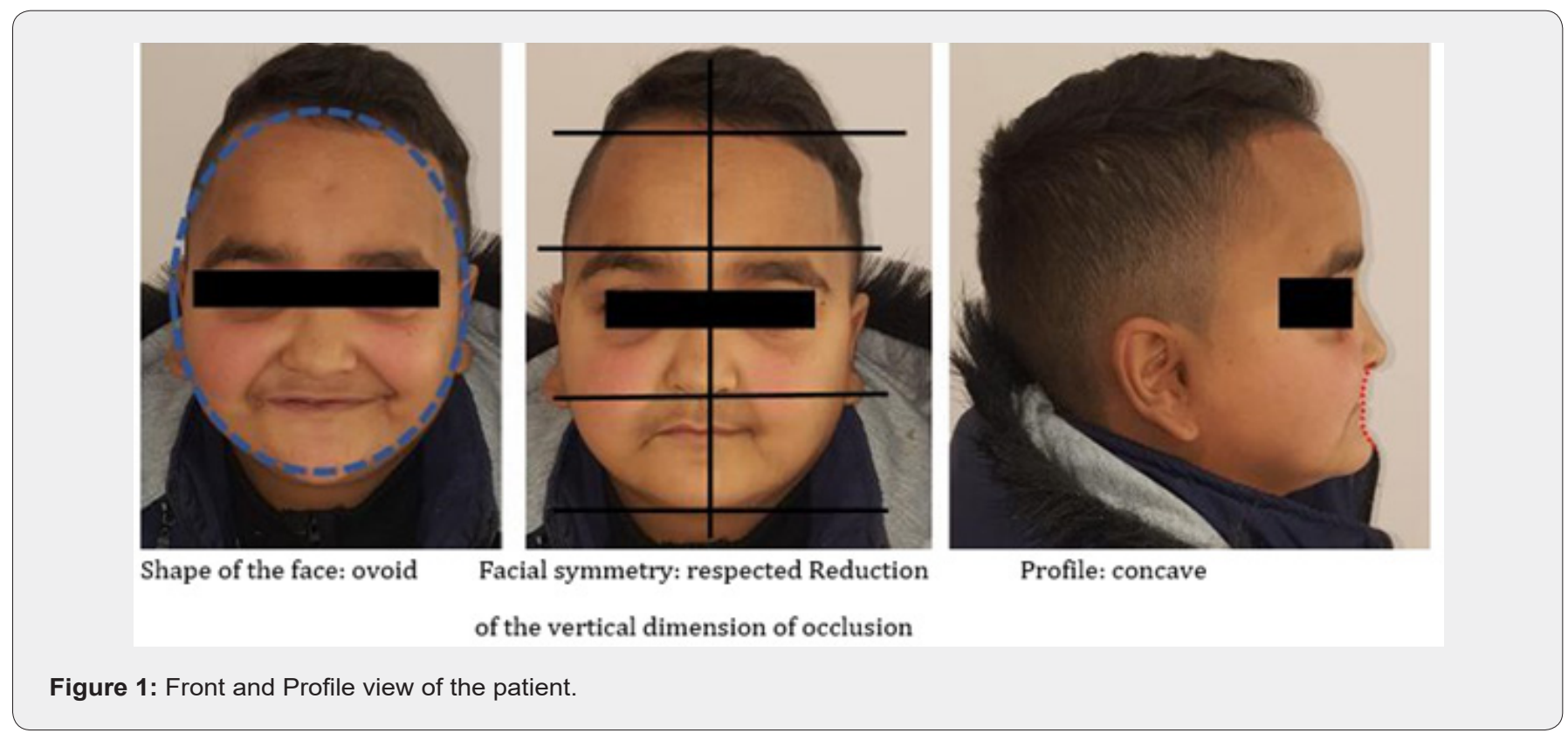

\section{Intraoral examination}

The intraoral examination of the maxillary arch shows that its entire mucosa presents canker sores. Its shape is parabolic, the set of ridges are resorbed however the right sector's resorption is more advanced than that of the left one, the median brake is absent the vestibule of the former sector is flat, the saliva is not very viscous and exists in small quantities, the Eisenring areas are shallow and the palate is flat. The entire maxillary mucosa presents canker sores. The intraoral examination of the mandibular arch reveals strongly resorbed arches with small trigons. The entire vestibule's depth, both in the maxillar and mandibular arch, is not exceeding $4 \mathrm{~mm}$ (Figure 2). Moreover, we note the existence of a jaw-like cicatricial bridle related to paracentesis; the patient has a severe microstomia (mouth opening amplitude is $30 \mathrm{~mm}$ ) (Figure 3). 


\section{Advances in Dentistry \& Oral Health}
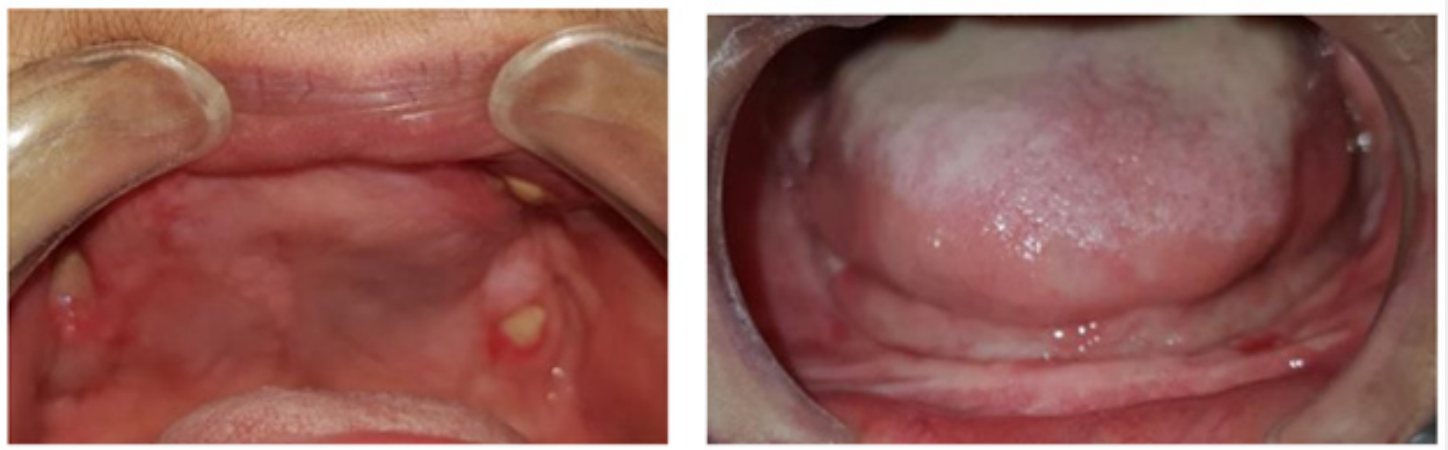

Figure 2: Intraoral view of the patient.

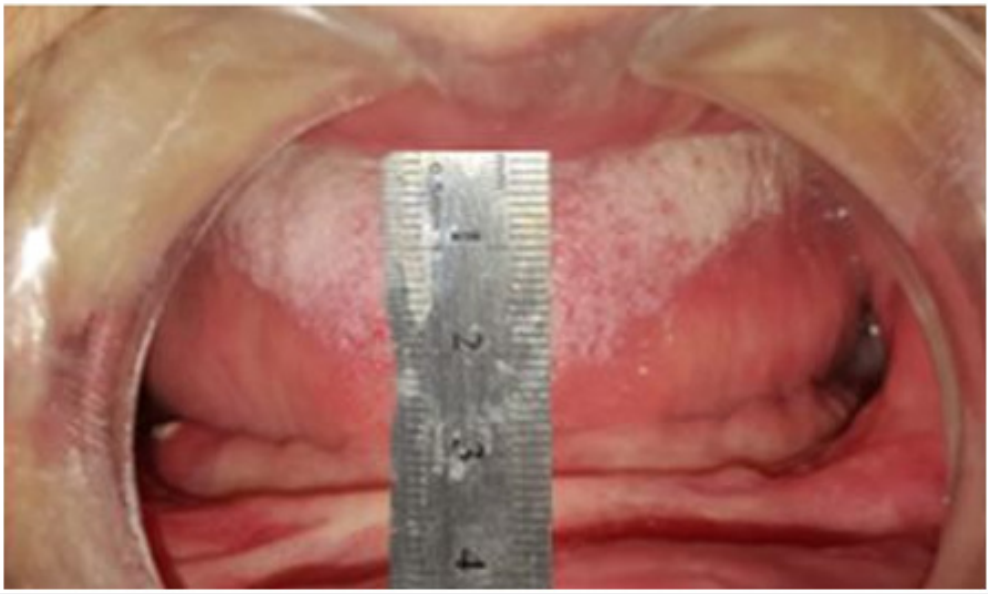

Figure 3: The amplitude of the patient's mouth opening testifies a severe microstomia $(30 \mathrm{~mm})$

\section{X-ray examination}

The radiological examination (Figure 4) shows that there are no residual teeth on the arches, bone resorption is more severe in the mandibular ridges.

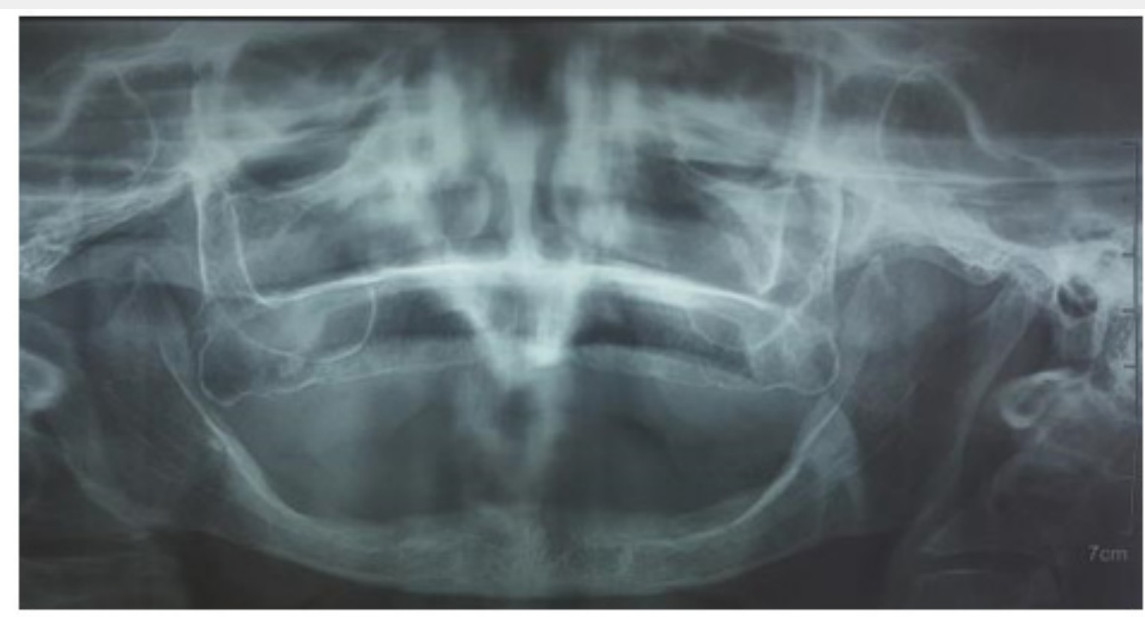

Figure 4: Panoramic x-ray of the patient. 


\section{Therapeutic goals}

i. Provide functional rehabilitation of all manducatory functions (phonation, mastication, and swallowing).

ii. Improve patient aesthetics.

iii. Achievement of these two goals will ensure social reintegration and improvement of the patient's self-esteem.

iv. The chosen therapeutic solution is a complete removable denture.

However, a set of obstacles emanating from this case made its management complex, namely:

a) Microstomia: Making the insertion and removal of various prosthetic devices (commercial and individual impression trays, wax occlusion rims) difficult and likely to injure the patient.

b) The general delicate state of the patient: The accumulation of a large number of pathologies (Hypofibrinogenemia, severe neutropenia on ALPS, and Myelosuppression) increases the risk of hemorrhage and infection of the patient, particularly during the acute phases when the patient hemogram is disturbed.

c) Intraoral conditions: Advanced resorption of the ridges (bimaxillary Classe III of Sanguiolo's classification), the shallow vestibule, and xerostomia associated with its low viscosity are likely to reduce the retentive potential of future dentures.

d) The status of the patient: Being educated in Ouazzane ( $361.7 \mathrm{~km}$ from Casablanca to 5 hours of travel) it was necessary to organize close care sessions, the time allocated was only 2 weeks, however with the help of a sibling living $106 \mathrm{~km}$ from Casablanca

(1h30 by car) the patient and his father were able to stay nearby which led us to organize the care sessions as follows:

The first appointment for the primary impressions, the next day at $2 \mathrm{pm}$ during which the functional secondary impressions were taken, then 3 days after the registration of the occlusion's rims and the choice of teeth, followed by the fitting of the teeth 3 days later and placing in the muffle the next day. The organization of close meetings was facilitated by the active participation of our dental technician at the D.C.T.C of Casablanca. Taking into account the medical history of the patient, the medical environment being familiar to him, and taking into account the psychological approach duly ensured by his pedodontist and perpetuated during the prosthetic phases, the patient was very cooperative and actively participated in the organization of his appointments.

\section{Primary impressions}

Pedodontic metal impression maxillary tray was tested and validated, the setting time was reduced by using warm water during mixing, however, the reduced quality of the mandibular impression (pulling, overextensions and gaps) led to a direct impression using a heavy putty silicone $\{$ Optosil@\}, the extrados was modeled so that it looks like an impression tray by adding a handle, this impression was relined by a silicone light to increase its accuracy. After disinfection, these impressions were sent for casting (Figure 5).

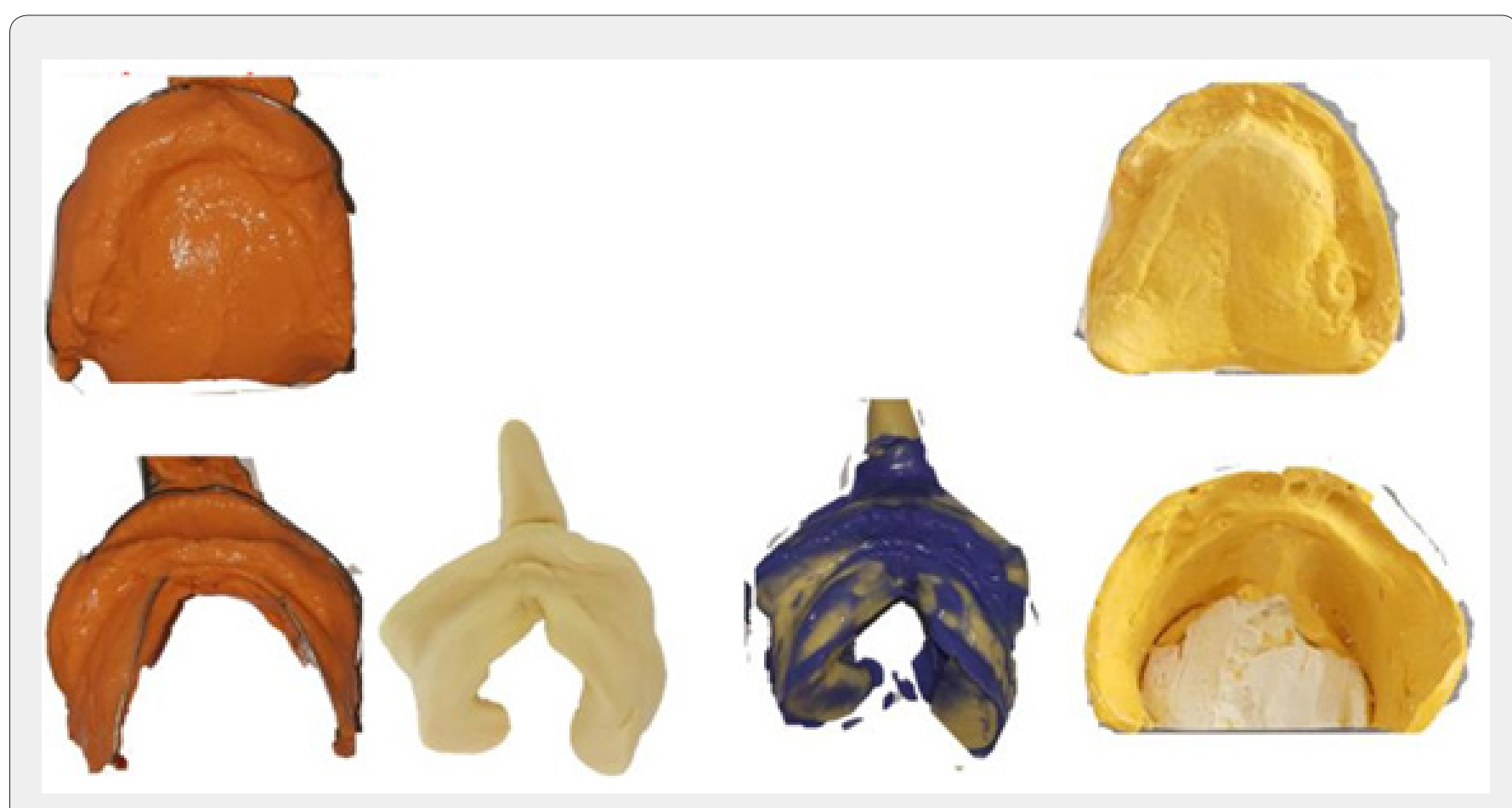

Figure 5: Primary impressions and plaster cast. 


\section{Functional secondary impressions}

A functional impression is made after static and dynamic checking of fit of the custom tray and its possible corrections. After static and dynamic checking of fit of the custom trays, maxillary Functional margins were formed with a thermoplastic material « Kerr® thermoplastic compound » under the patient's active movements; The Second functional impression was made by paste based on eugenol's zinc oxide "Impression Past $₫$ ", The choice of material was dictated by the membrane's mucosal condition (the maxilla being free of inflammation or mouth ulcers, In contrast, given the persistence of canker sores in the mandible and given the advanced mandibular resorption we chose a Thiocol" Permlastic Regular ${ }^{\circledR}$ “ for Phonetic functional impression (Figure 6).
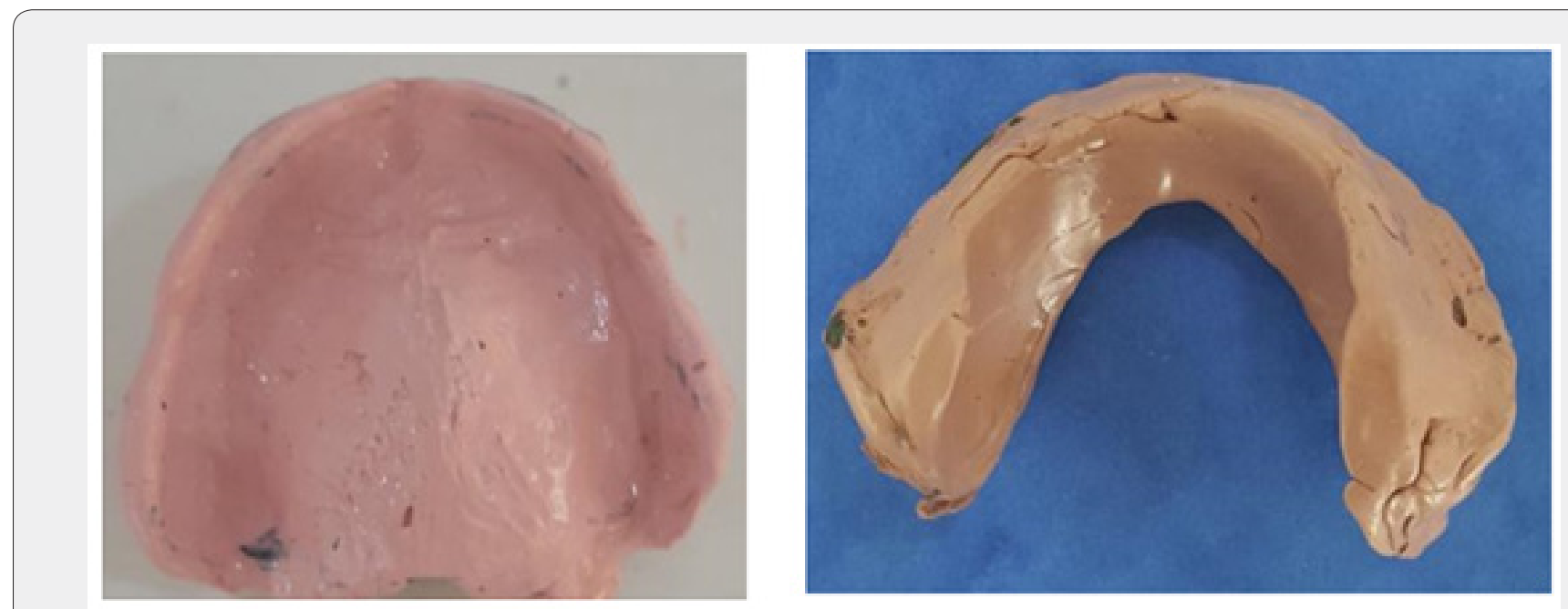

Figure 6: Functional secondary impressions.

\section{The record of intermaxillary relations}
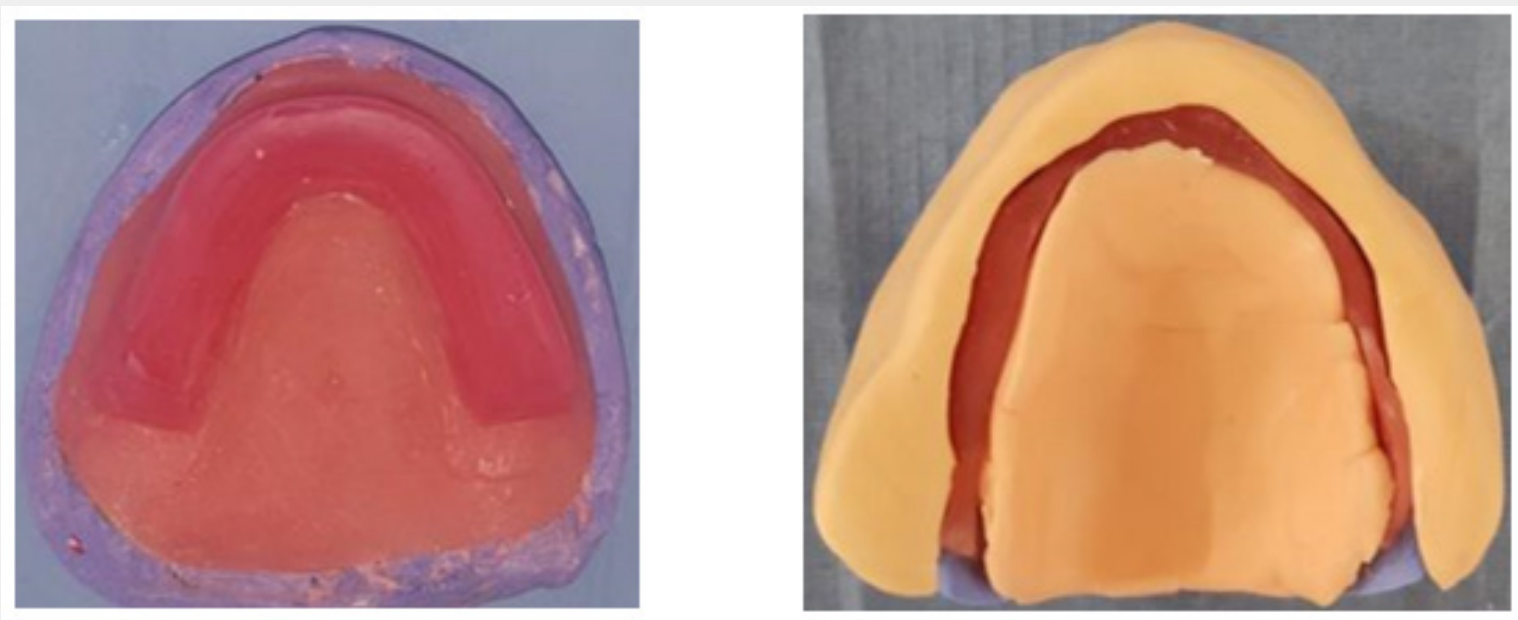

Figure 7: Wax occlusal rims.

After boxing the functional secondary impressions and their casting, two wax models were made (Figure 7).

The recording of jaw relations is more complicated in children and adolescents compared to adults:

First, the registration of the centric relationship among these young patients is difficult to achieve, then the parallelism required to adjust the occlusal plane is only possible through instruments (Fox plan) adapted to infantile mouth proportions. To this complexity are added the peculiarities relating to our patient: the concave profile must be corrected by ensuring good labial support, the vertical dimension must be reestablished harmoniously, the severe microstomia making the use of existing Fox plans (including a Pedodontic one) in our department impossible and requiring 
the making of a new adequate instrument. Thus, we tried the existing pediatric Fox plan of Fox, however, it was found that the distance between the occlusal tray and the horizontal outer edge of this instrument $(83 \mathrm{~mm})$ exceeded the maximum limits of our patient, moreover, the width of the occlusal tray being very small did not correspond to the volume of the maxillary occlusion rim compatible with a harmonious labial and jugular support (Figure 8).

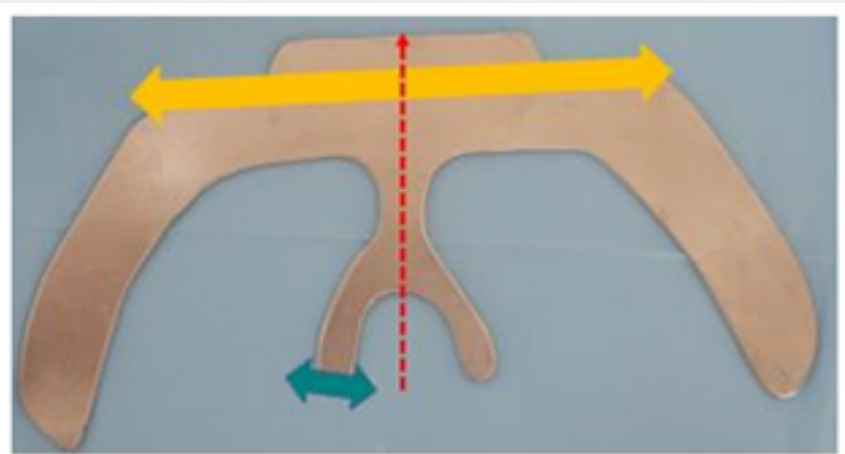

Pediatric Fox plan

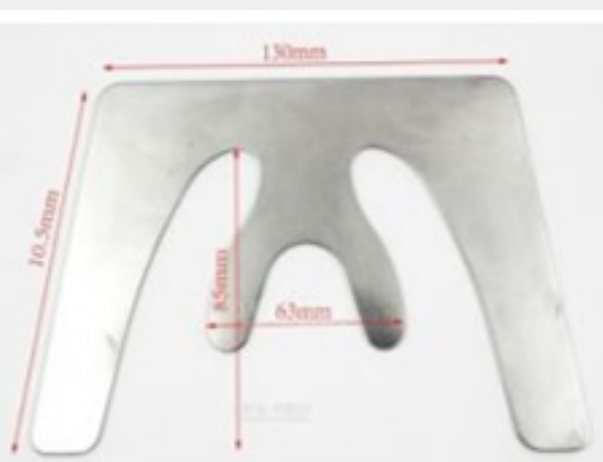

Fox plan for adult

Figure 8: Image showing poor adaptation of different Fox planes to patient's intraoral dimensions.

\section{The computer-aided design of the pediatric Fox Plan}

After collecting the necessary information including the width of the patient's face, the distance between the nasal point of the middle of the tragus, the depth of the maxillary arch and the width of the maxillary occlusion rim, we started the 3D design of the FOX plan using Meshmixer® software by importing an STL file (stereolithographic) designed based on the general shape of a handle holding the dental floss, we created the occlusal tray (L $=56 \mathrm{~mm} \mathrm{l}=54 \mathrm{~mm} \mathrm{E}=2.50 \mathrm{~mm}$ ) to which, we added a horizontal rectangle $(\mathrm{L}=84 \mathrm{~mm}, \mathrm{l}=5.3 \mathrm{~mm}$ and $\mathrm{E}=2.50 \mathrm{~mm})$ and two lateral strangles $(\mathrm{L}=64 \mathrm{~mm} \mathrm{l}=53 \mathrm{~mm} \mathrm{E}=2.50 \mathrm{~mm}$ ) forming each An angle of $110^{\circ}$ with the horizontal branch, the following figure shows the general shape of the designer Fox plan (Figure 9).

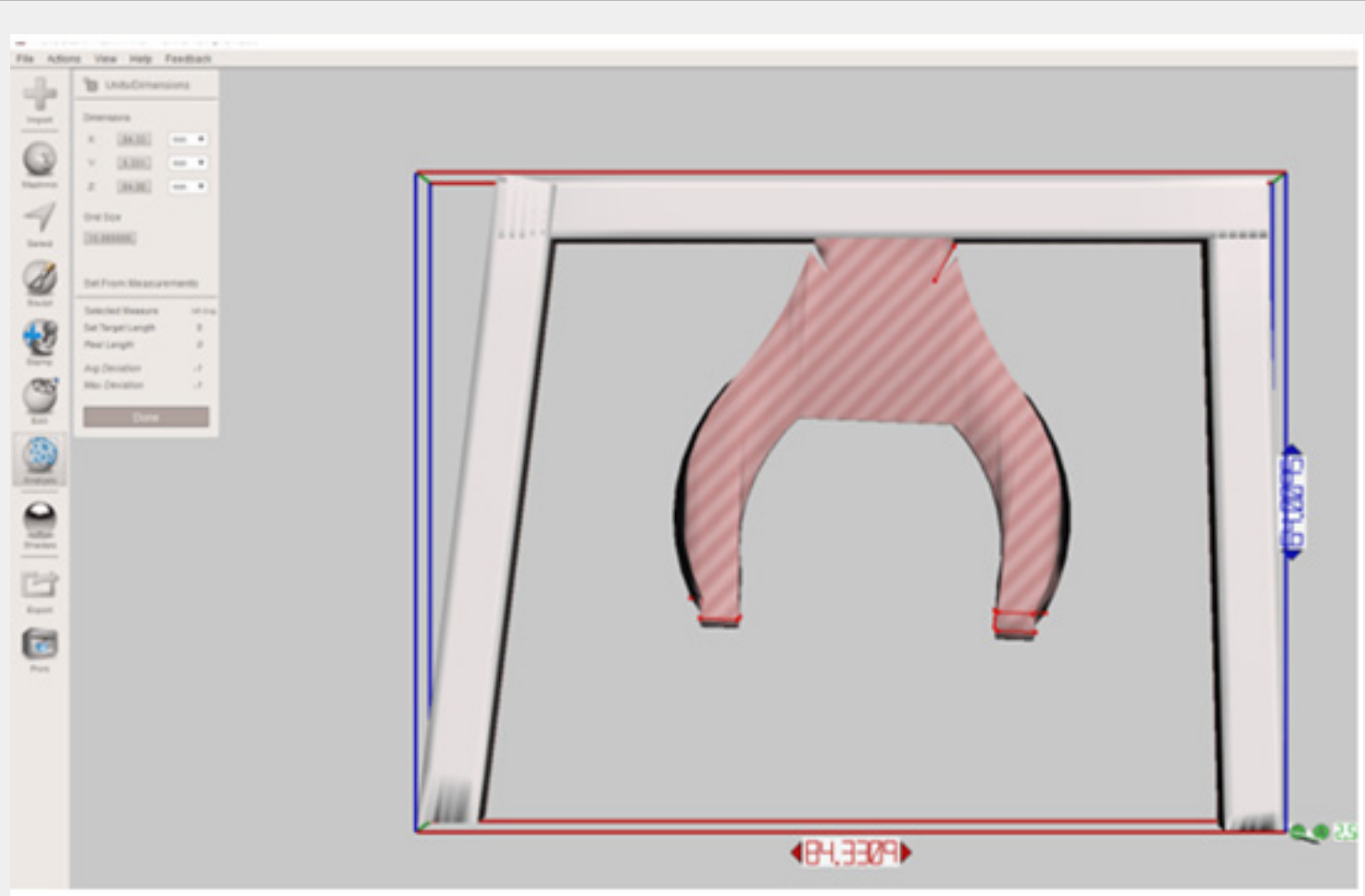

Figure 9: digital design of the Fox plan \{software:MeshMixer®\}. 


\section{D printing of the Fox plan}

After designing the Fox plan, its STL file was imported into the "XYZ ware®" software compatible with our "Da Vinci Junior" 3D printer from the company "XYZ", in this software, we added vertical supports and a rectangular platform (Figure 10), the printing time was about $1 \mathrm{~h} 03 \mathrm{~min}$ (Figure 11). The 3D printer works by the fused deposition modeling of a thermoplastic material which was in this case a filament of PLA (polylactic acid). Polylactic and polylactide acid are often confused because they have the same repetitive unit. The difference between the two terms is that polylactic acid is synthesized directly from lactic acid whereas polylactide is produced from lactide. The most widely used PLA synthesis method is the ring-opening polymerization of lactide. It is used as a spool of thread which is driven to the extrusion head where the PLA filaments are melted and deposited in fine drops on the printer tray. This material is translucent in its natural form but allows one to make objects of different colors using reels of son tinted in the mass. Its melting temperature is $150^{\circ} \mathrm{C}$ and the thickness of the imprinted printed layers varies approximately between 70 and 400 microns (300 microns in our case) [4].

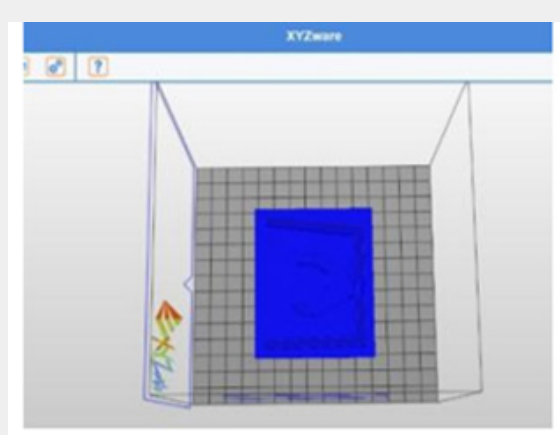

Figure 10: Fox Plan Preparation for 3D Printing $\{X Y Z W a r e ®\}$.

As part of the "Open Source" community where sharing is recommended, we made the STL file accessible by posting it on www.Thingivers.com (Figure 11). The figure above shows different views of the FOX plane after printing and removing support rods (Figure 12). Once the fox plan was adapted to the patient, we proceeded first to the restoration of harmonious labial support by acting on the angulation volume and the height of the anterior occlusion bead, then thanks to this new instrument we adjusted the occlusion plane conventionally by looking for parallelism with the bi-pupillary line anteriorly and posteriorly to camper plan (Figure 13). Adjustment of the vertical dimension was performed based on the vertical physiological rest dimension (VPRD) and the aesthetic rendering in the patient. The recording of the centric relation (CR) was made by the wax "Aluwax $\AA$ " after having validated that the $\mathrm{CR}$ is reiterative and reproducible (Figure 14).

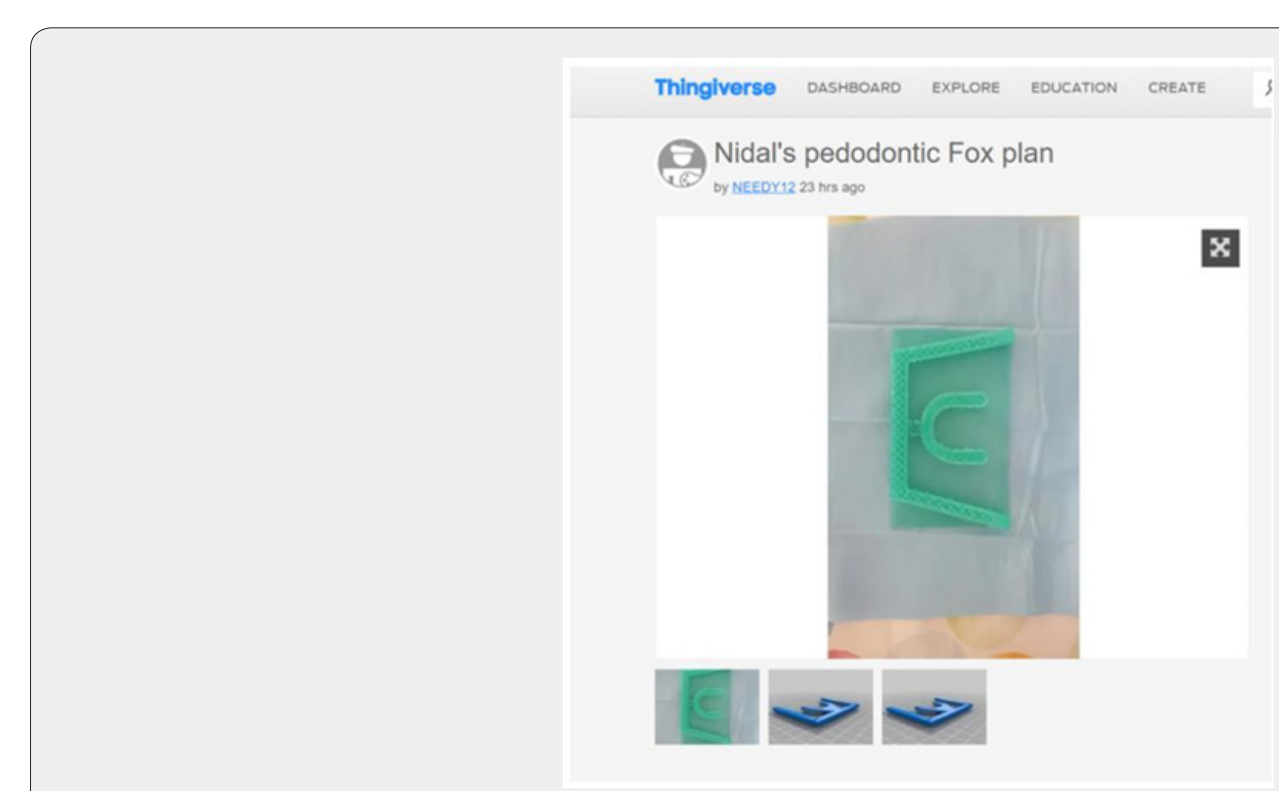

Figure 11: publication of the Fox plan's STL file on the "Thingivers" website. 


\section{Advances in Dentistry \& Oral Health}
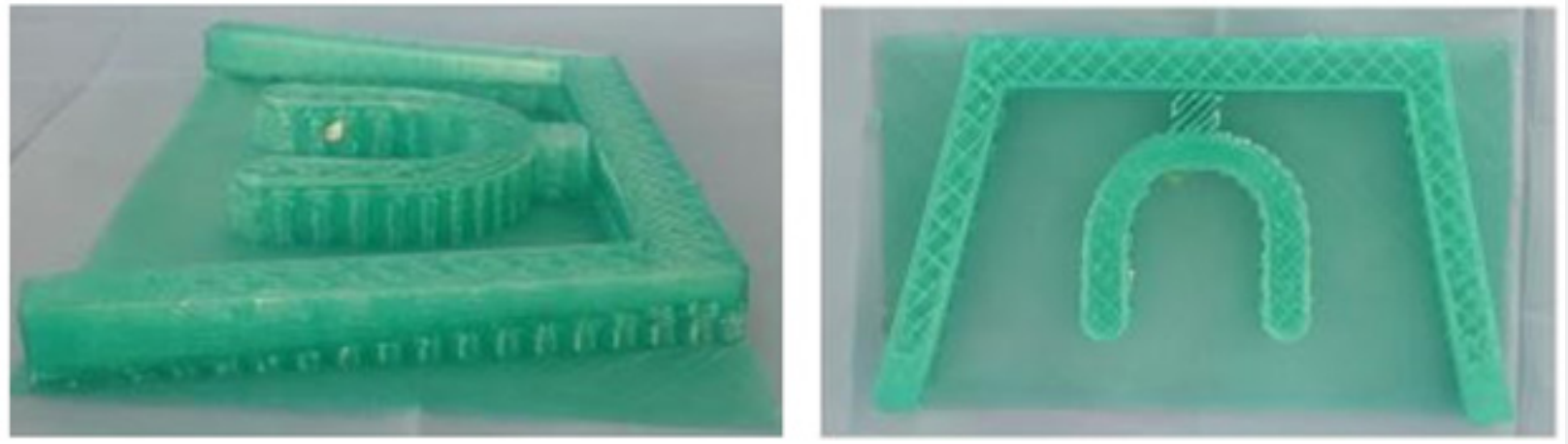

Figure 12: Fox plan printed in PLA (polylactic acid).
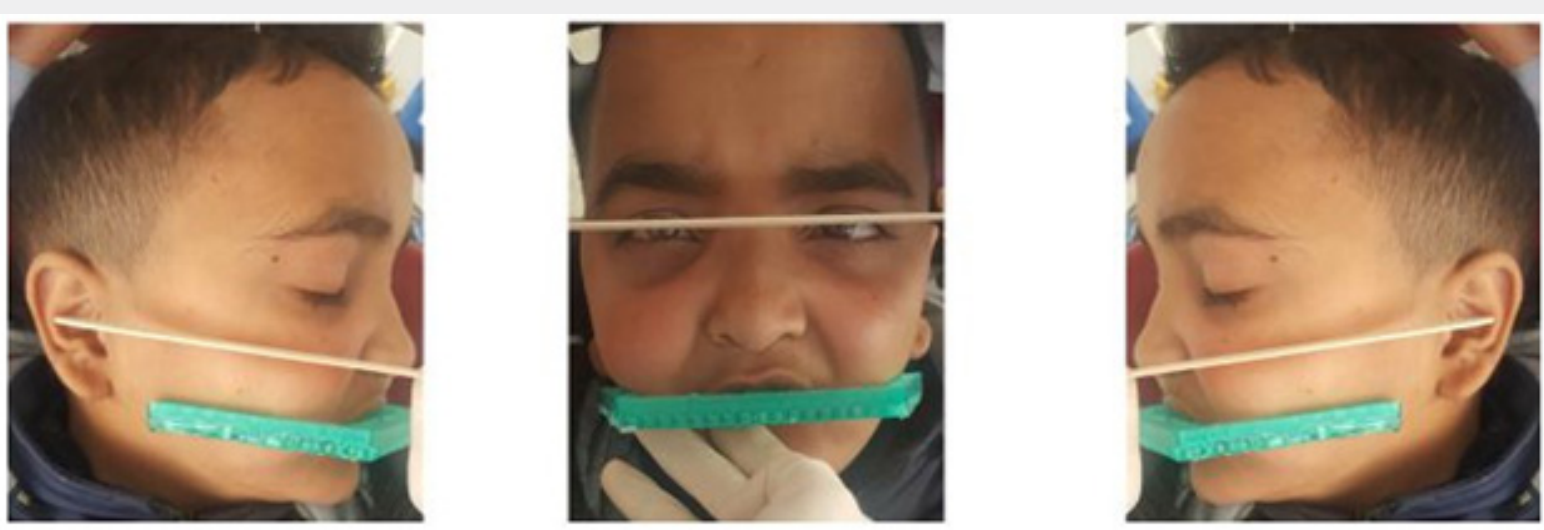

Figure 13: Adjustment of the occlusal plan.

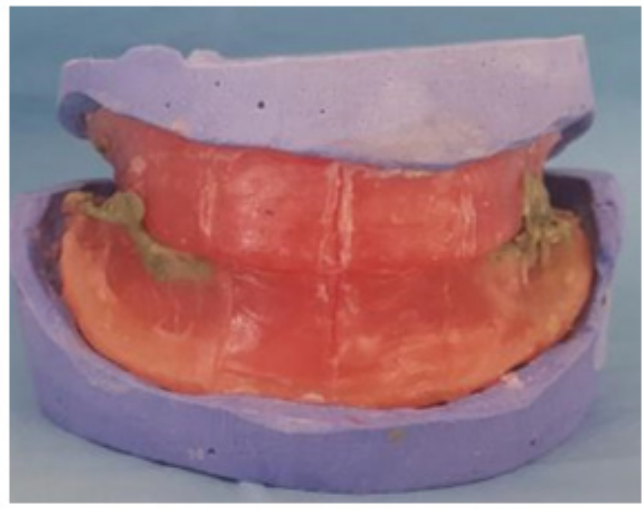

Figure 14: Centric relation recording.

\section{Teeth choice}

Based on the inter-canine distance $(35 \mathrm{~mm})$ and the height of the anterior bead $(8 \mathrm{~mm})$ we have chosen a set of complete bimaxillary teeth corresponding to its measurements, the shade is A2. Given the reduced width of the jaws, we opted for a shortened arch avoiding the mounting of the second molars (Figure 15).

The teeth fitting has allowed their aesthetic and functional validation both by us (practitioner) and by the patient himself (Figure 16). 


\section{Advances in Dentistry \& Oral Health}
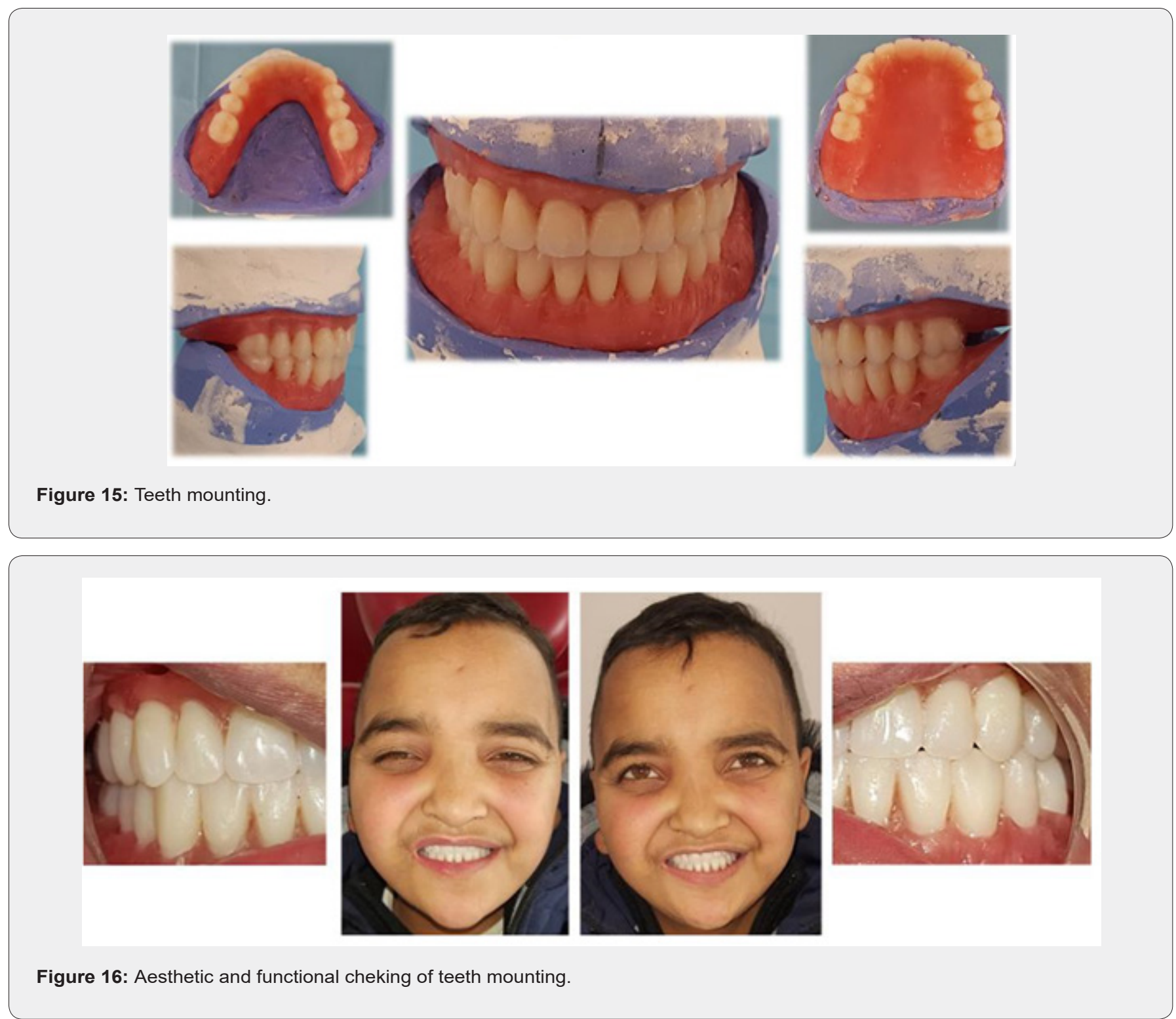

\section{Denture insertion}

After the muffling and polishing steps, prostheses were placed in the mouth with insisting on post-prosthetic advice, such as the prohibition of sleeping with the dentures on, their regular cleaning and scheduling control appointments concording with the ENT and cardiologist appointments. We also insisted on the introduction of foods with a soft consistency at the beginning and then a harder consistency gradually. Given the general condition of the patient, we warned him of any inflammation or bleeding that could alter his hemodynamic balance. despite the reduced growth potential in this patient we planned second dentures activated by transverse and anteroposterior expansion's artifices on a bi-annual average and the generated space would be filled by a chemo-polymerizable resin such as "Rebarond ${ }^{\circledR}$ " this activation must be monitored by the treating dentist, and this is how the dentures were duplicated to allow the patient to leave with functional prosthesis while we continue the realization of the evolutionary ones.

\section{Discussion}

Because of the loss of teeth, all the manducatory functions are affected: phonation, swallowing and mastication, which generates several disorders and parafunction that affect the physical growth and maturation of the patient, in fact, the alteration of aesthetics degrades the image of the child of himself, especially as the infant environment to which he adheres (friends, neighbors and classmates) does not assimilate the early loss of teeth making schooling of these young patients difficult. As a result, the dentist participates not only as such but also as a therapist to facilitate the integration of the new prosthesis. 
Prosthetic management in children and adolescents is complicated by the reduction of the perimeter of the mouth, the importance of the psychological approach to facilitate the patient/ practitioner exchange, but also the high esthetic expectations of the patients. the case of our patient where the alteration of the general state affects the oral anatomical elements. As part of stabilization, sustentation, and retention, the realization of a pediatric prosthesis becomes a real challenge [5]. Growth management is a parameter to be taken care of as well, in fact, to realize good prostheses that will last only 6 months is derisory, the planning and the addition of artifices participating at the same time to accompany the growth of the arches (in our case we have planned to realize a Quadhelix®) can actively support the patient. The post-prosthetic check appointments are essential and can highlight any grievance early so as not to disrupt the acceptance of the prosthesis by the patient.

\section{Conclusion}

The loss of teeth in children causes the distortion of self-image loss of self-confidence which subsequently affects the balance of their psychomotor and will make difficult their schooling. Prosthetic rehabilitation will not only restore function and aesthetics but will repair the after-effects caused by edentulism.

\section{References}

1. Shah S, Wu E, Rao VK (2014) Autoimmune Lymphoproliferative Syndrome: An Update and Review of the Literature. Curr Allergy Asthma Rep 14(9): 460-462.

2. Lambotte O, Neven B, Galicier L, Magerus Chatinet A, Schleinitz N, et al. (2013) Diagnosis of autoimmune lymphoproliferative syndrome caused by FAS deficiency in adults. Haematol 98(3): 389-392

3. Liébart MF, Fouque-Deruelle C, Santini A (2004) Smile line and periodontium visibility. Perio 1(1): 17-25.

4. Gamez Perez J, Velazquez Infante JC, Franco Urquiza E, Pages P, Carrasco F, et al. (2011) Fracture behavior of quenched poly (lactic acid). Express Polymer Letters 5(1): 82-91.

5. Siepmann S, Heydecke G, Holst S, Holst AL (2008) Technical treatment considerations in making prostheses for children. Child prostheses. Rev Mens Suisse Odontostomatol 118(12): 1177-1186.

\begin{tabular}{l} 
Your next submission with Juniper Publishers \\
will reach you the below assets \\
- Quality Editorial service \\
- Swift Peer Review \\
- Reprints availability \\
- E-prints Service \\
- Manuscript Podcast for convenient understanding \\
- Global attainment for your research \\
- Manuscript accessibility in different formats \\
( Pdf, E-pub, Full Text, Audio) \\
- Unceasing customer service \\
Track the below URL for one-step submission \\
https://juniperpublishers.com/online-submission.php \\
\hline
\end{tabular}

